\title{
Ophthalmic services in Shanghai 2017: a cataract-centric city-wide government survey
}

Xiangjia Zhu ${ }^{1,2 \dagger}$, Yu Du ${ }^{1 \dagger}$, Wenwen He ${ }^{1 \dagger}$, Jinhui Dai ${ }^{1 \dagger}$, Minjie Chen ${ }^{1}$, Peijun Yao ${ }^{1}$, Han Chen ${ }^{1}$, Hui Ren ${ }^{1}$, Yuan Fang ${ }^{1}$, Shensheng $\operatorname{Tan}^{2}$ and $Y i L^{1,2^{*}}$ (D)

\begin{abstract}
Background: Demand for eye care has increased in recent decades in China due to rapid socioeconomic development and demographic shift. Knowledge of output and productivity of ophthalmic services would allow policymakers to optimize resource allocation, and is therefore essential. This study sought to map the landscape of ophthalmic services available in Shanghai, China.

Methods: In 2018, a government-led survey was conducted of all 86 tertiary/secondary hospitals and five major private hospitals providing eye care in Shanghai in the form of electronic questionnaire, which encompassed ophthalmic services (outpatient and emergency room [ER] visit, inpatient admissions, and surgical volume) and service productivity in terms of annual outpatient and ER visits per doctor, inpatient admissions per bed, and surgical volume per doctor. Comparisons were made among different levels of hospitals with categorical variables tested by Chi-square analysis.

Results: The response rate was $85.7 \%$. The Eye and Ear, Nose, and Throat (EENT) Hospital was the largest tertiary specialty hospital, and alone contributed to the highest $21.0 \%$ of annual ophthalmic outpatient and ER visits (visits per doctor: 5460), compared with other 26 tertiary hospitals, 46 secondary hospitals and five private hospitals (visits per doctor: 3683, 4651 and 1876). The annual inpatient admission was 20,103,56,992, 14,090, and 52,047 for the EENT Hospital, all the other tertiary hospitals, secondary hospitals and five private hospitals, respectively. Turnover rates were highest for the EENT Hospital and private hospitals. The average surgical volume at the EENT Hospital was 72,666, exceeding that of private $(15,874.8)$ and other tertiary hospitals (3366.7). The EENT Hospital and private hospitals performed 16,982 (14.2\%) and 55,538 (46.6\%) of all cataract surgeries. Proportions of both complicated cataractous cases and complicated cataract surgeries at the EENT Hospital was the highest, followed by other tertiary and secondary/private hospitals $(P<0.0001)$.
\end{abstract}

\footnotetext{
* Correspondence: luyieent@163.com

${ }^{\dagger}$ Xiangjia Zhu, Yu Du, Wenwen He and Jinhui Dai are Co-first authors.

${ }^{1}$ Eye Institute of Eye and Ear, Nose, and Throat Hospital of Fudan University;

Key Laboratory of Myopia, Ministry of Health; Key Laboratory of Visual

Impairment and Restoration of Shanghai, Fudan University; Key NHC key

Laboratory of Myopia, Fudan University; Chinese Academy of Medical

Sciences, 83 Fenyang Road, Shanghai 200031, China

${ }^{2}$ Shanghai Medical Quality Control Management Center, 1477 West Beijing

Road, Shanghai 200040, China
}

(c) The Author(s). 2021 Open Access This article is licensed under a Creative Commons Attribution 4.0 International License, which permits use, sharing, adaptation, distribution and reproduction in any medium or format, as long as you give appropriate credit to the original author(s) and the source, provide a link to the Creative Commons licence, and indicate if changes were made. The images or other third party material in this article are included in the article's Creative Commons licence, unless indicated otherwise in a credit line to the material. If material is not included in the article's Creative Commons licence and your intended use is not permitted by statutory regulation or exceeds the permitted use, you will need to obtain permission directly from the copyright holder. To view a copy of this licence, visit http://creativecommons.org/licenses/by/4.0/ The Creative Commons Public Domain Dedication waiver (http://creativecommons.org/publicdomain/zero/1.0/) applies to the data made available in this article, unless otherwise stated in a credit line to the data. 
Conclusions: In Shanghai, public providers dominate ophthalmic services especially for complicated cases, with almost one fifth of services provided by the EENT Hospital alone, while private sectors, though not large in number, still effectively help meet large proportions of eye care demand. Optimization of hierarchical medical system is warranted to improve the efficiency and standardization of ophthalmic services.

Keywords: Ophthalmic service, Cataract surgery, Public hospital, Private hospital, China

\section{Background}

China, as the most populous country in the world, has achieved remarkable progress in transitioning from a centrally planned economy towards a market-oriented economy over the last 40 years. However, the efficiency and equity of China's healthcare system, which potentially affects the lives of nearly 1.40 billion people, have developed unsatisfactorily $[1,2]$. With the rapid demographic shift towards an aging population and the simultaneous epidemiological change of the disease burden into predominantly non-communicable diseases, especially age-related diseases, it is imperative for China to revamp the existing healthcare delivery system to meet the increasing demand for medical services [3].

Despite rapid development, public hospitals are struggling to cater for the rising numbers of patients. Therefore, the government has implemented numerous favorable policies to facilitate the growth of private services to expand access to healthcare and to alleviate the burden of healthcare delivery $[4,5]$. In 1980, China first issued a policy that encouraged qualified medical professionals to engage in private practice [6]. In 2010, the State Council published a policy document that further encouraged social capital investment in healthcare [7]. By 2017, the number of private hospitals in China had increased to 447,160 [8], and these hospitals address the shortage of public healthcare services. Nevertheless, careful attention should be given to the efficiency of hospitals to evaluate their performance and to improve the coordination of different sectors.

Impaired vision is a high-priority public health issue, and population aging in China is causing a dramatic increase in the burden of age-related ocular diseases, such as cataracts and macular degeneration [9-11]. To improve eye care services in China, data are needed on the current availability and use of ophthalmic services. Shanghai is the economic center and the second most populous city in China, and faces tough challenges associated with an aging society. In 2016, $13.0 \%$ of the registered population was over 65 years old, a percentage that increased to $21.8 \%$ in 2017 according to data provided by the Shanghai Municipal Statistics Bureau.

Considering these data, the future demand for eye care services is likely to outpace the available resources. Thus, periodic assessment of the availability and productivity of ophthalmic services in Shanghai during the current period of healthcare reform could be very meaningful. However, few studies have addressed this issue to date.

In 2018, the Shanghai Quality Control Center of Clinical Ophthalmology, an evidence-based center that conducts clinical care evaluations of ophthalmology, launched the first electronic questionnaire survey to obtain baseline information about the characteristics of eye care providers and their practices in Shanghai. The survey investigated the distribution of the ophthalmic services across different subspecialties. It paid particular attention to cataract surgery because it was classified as the prioritized eye disease to be addressed in China in the 13th 5-Year National Plan of Eye Health launched by the National Health Commission in 2016.

\section{Methods}

This was an observational, cross-sectional, questionnaire survey of hospitals providing specialty eye care in Shanghai. This study involved the analysis of data acquired from a government-led questionnaire survey, which did not involve any human participants, personal identifiable information or animal experiments. Thus, according to the Ethics Committee at the EENT Hospital of Fudan University, this study involved no ethical issues, and was reviewed and deemed exempt from formal ethics approval or consent forms from the participating hospitals. Via emails or telephone calls with the administrative offices at the target hospitals, we explained the purpose of the survey and asked for their participation. Only those who agreed to participate completed the questionnaire.

\section{Participating hospitals}

We considered it essential to collect data from all 86 tertiary and secondary hospitals with an ophthalmology department and from five major high-volume private eye hospitals.

\section{Questionnaire}

The questionnaire (Supplementary file) was developed by a panel of eye doctors in Shanghai based on previous published reports of other countries and was sent to the eligible hospitals in 2018 to collect information of ophthalmic services for the 2017 fiscal year. Data collection was completed in October 2018. The questionnaire had two main parts: the basic characteristics of the hospital and the level of ophthalmic specialty services, which 
included (1) outpatient and inpatient services, (2) professional staff, (3) ophthalmic equipment, (4) volumes of different types of ophthalmic procedures, and (5) specific data on cataract surgery. Since the questionnaire used in this survey only included data collection of objective subjects without any subjective judgement, validation was not necessary.

The questionnaires were sent by standard mail to all eligible hospitals by the Shanghai Quality-control Center of Clinical Ophthalmology. The correspondence included a clear explanation of the survey's purpose and the questionnaires were to be completed by an appropriate person in the administrative office at each hospital to ensure accuracy. Deadline was October 2018 with a reminder email sent to the hospitals if they did not return the questionnaire.

\section{Measures and analysis}

The responses to the questions were summarized as proportions or means. Hospital output was assessed in terms of the number of outpatient and emergency room (ER) visits, the number of the inpatient admissions, and surgical volume. Service efficiency was measured as the annual outpatient and ER visits per doctor, annual inpatient admissions per bed, and the annual surgical volume per doctor. Information pertaining to the demographic data of Shanghai in 2017 was extracted from China's Health Statistics Yearbook 2018. Comparisons among categorical variables among different levels of hospital were conducted by Chi-square analysis (Prism Version 9.9.1, GraphPad Software, LLC).

Among the tertiary hospitals surveyed in this study, one stood out, the EENT Hospital of Fudan University, as the largest hospital specialized in ophthalmology and otolaryngology in China. The EENT Hospital treats a much greater number of ophthalmic patients than other hospitals in Shanghai. Therefore, its data were analyzed separately from those of other tertiary hospitals.

\section{Results}

\section{Participating hospitals}

Among 91 eligible hospitals, 78 hospitals responded (response rate of $85.7 \%$ ), of which 27 were tertiary hospitals (EENT Hospital plus 26 other tertiary hospitals), 46 were secondary hospitals, and five were major high-volume private hospitals. All the tertiary hospitals and private hospitals responded while 13 secondary hospitals did not.

\section{Basic characteristics of the hospitals: professionals, hospital beds, and equipment}

In 2017, there were over 3000 doctors, nurses and technicians providing eye care in Shanghai, representing approximately 0.49 ophthalmologists per 10,000 population. Overall, $83.7 \%$ of eye doctors worked in public hospitals, which included $14.8 \%$ at the EENT Hospital and $47.8 \%$ at the other 26 tertiary hospitals (Table 1). The mean number of eye doctors with senior titles (chief physician and associate chief physician) was greatest at the EENT Hospital (67), followed by private hospitals (19.6), other tertiary hospitals (8.0), and secondary hospitals (2.0).

The survey recorded 1492 beds for hospitalized ophthalmic patients in Shanghai in 2017. Of these, 10.1, 50.7, and $16.2 \%$ were at the EENT Hospital, other tertiary hospitals, and secondary hospitals, while the five private hospitals had $23.1 \%$ of beds. On average, the number of beds at the EENT Hospital (150) exceeded the mean number of beds at private hospitals (68.8), tertiary hospitals (29.1), and secondary hospitals (5.2).

In terms of the distribution of medical personnel among hospitals, the EENT Hospital was the best equipped, with the most comprehensive ophthalmic examinations available, followed by private hospitals (Table 2). Unsurprisingly, the secondary hospitals lacked complex or advanced equipment, such as fluorescence angiography, confocal microscopy, vitrectomy instruments, and femtosecond lasers, compared with tertiary hospitals.

Table 1 Total number and composition of professional and allied staff at each hospital level

\begin{tabular}{|c|c|c|c|c|c|c|c|c|c|}
\hline Staff Number & $\begin{array}{l}\text { Chief } \\
\text { Physician }\end{array}$ & $\begin{array}{l}\text { Associate Chief } \\
\text { Physician }\end{array}$ & $\begin{array}{l}\text { Attending } \\
\text { Physician }\end{array}$ & Resident & $\begin{array}{l}\text { Ophthalmic } \\
\text { Nurse }\end{array}$ & $\begin{array}{l}\text { Theater } \\
\text { Nurse }\end{array}$ & $\begin{array}{l}\text { Ordinary } \\
\text { Nurse }\end{array}$ & Optometrist & Total \\
\hline EENT Hospital & 28 & 39 & 39 & 70 & 145 & 109 & 36 & 20 & 486 \\
\hline $\begin{array}{l}\text { Other tertiary } \\
\text { hospitals }\end{array}$ & $95(3.7)$ & $113(4.3)$ & $150(5.8)$ & 209 (8.0) & 275 (10.6) & $97(3.7)$ & $178(6.8)$ & $112(4.3)$ & $\begin{array}{l}1229 \\
(47.3)\end{array}$ \\
\hline $\begin{array}{l}\text { Secondary } \\
\text { hospitals }\end{array}$ & $44(1.0)$ & $47(1.0)$ & $115(2.5)$ & $44(1.0)$ & $131(2.8)$ & $56(1.2)$ & $75(1.6)$ & $41(0.9)$ & $\begin{array}{l}553 \\
(12.0)\end{array}$ \\
\hline Private hospitals & $57(11.4)$ & $41(8.2)$ & $43(8.6)$ & $52(10.4)$ & $263(52.6)$ & $56(11.2)$ & $207(41.4)$ & $41(8.2)$ & $\begin{array}{l}760 \\
(152.0)\end{array}$ \\
\hline Total & 224 & 240 & 347 & 375 & 814 & 318 & 496 & 214 & 3028 \\
\hline
\end{tabular}

Numbers in brackets represent the mean number of staff at each hospital level.

EENT Hospital Eye and Ear, Nose, and Throat Hospital of Fudan University 
Table 2 Availability of ophthalmic equipment at each hospital level

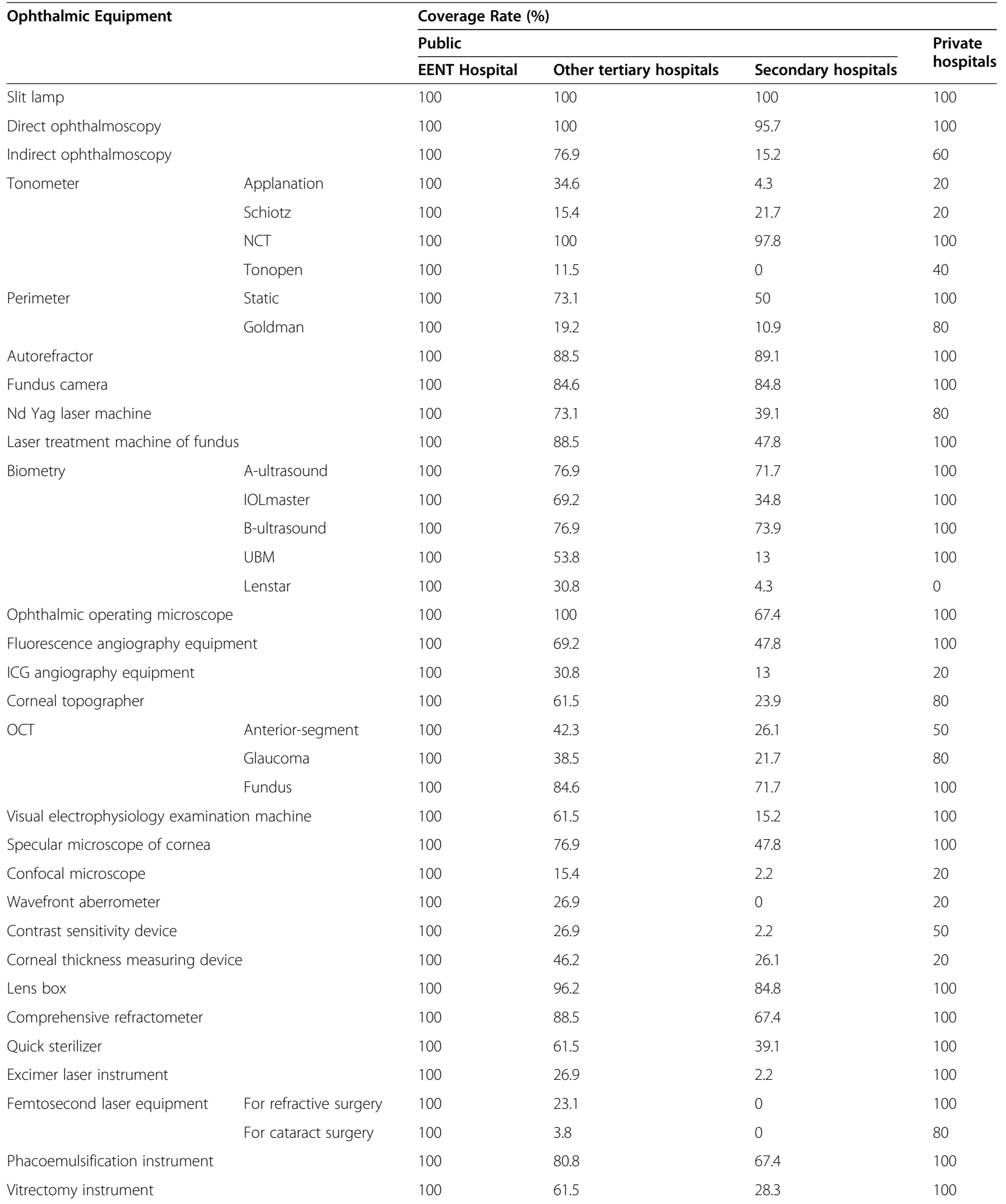

EENT Hospital Eye and Ear, Nose, and Throat Hospital of Fudan University, NCT non-contact tonometer, Nd Yag neodymium-doped yttrium aluminum garnet, UBM ultrasound biomicroscope, ICG indocyanine green, OCT optical coherence tomography 


\section{Ophthalmic service output and productivity}

The output of ophthalmic services was evaluated in terms of the number of outpatient and ER visits, the number of inpatient admissions, and surgical volume.

The annual number of ophthalmic outpatient and ER visits in 2017 was 4,574,143, of which $21.0 \%$ were provided by the EENT Hospital alone and $45.7 \%$ by other tertiary hospitals (Table 3). Private hospitals accounted for just $7.9 \%$ of the total number, which was relatively low considering they accounted for $16.3 \%$ of the total number of eye doctors. Generally, 31.7\% of outpatient and ER visits in Shanghai were due to non-local patients. This percentage was the highest at the EENT Hospital (61.0\%) followed by other tertiary hospitals (30.6\%), whereas secondary and private hospitals mostly served local patients (over $80 \%)(P<0.0001$, Chi-square analysis). Regarding the productivity of outpatient services, the number of outpatient and ER visits per eye doctor varied considerably among hospitals: 5460 at the EENT Hospital, 3683 at other tertiary hospitals, and only 1876 at private hospitals.

The total number of ophthalmic inpatient admissions in Shanghai was 143,232, of which $14 \%$ were at the EENT Hospital, $39.8 \%$ at other tertiary hospitals, and $36.3 \%$ at private hospitals (Table 3). The turnover rate, measured as the annual inpatient admissions per bed, was higher at the EENT Hospital and private hospitals (134 and 151, respectively) than at other tertiary hospitals and secondary hospitals (75 and 58, respectively).

Ophthalmic procedures are another major aspect of eye care provided to the public. A total of 265,365 ophthalmic procedures were performed in 2017. The EENT Hospital, other tertiary hospitals, and the private hospitals each provided about $30 \%$ of the total surgical volume (Table 3). On average, the EENT Hospital was ranked first in terms of the annual surgical volume, having performed 72,666 procedures, which greatly exceeded that of private hospitals $(15,874.8)$ and other tertiary hospitals (3366.7). The annual surgical volume per doctor was also greatest at the EENT Hospital (412.9), followed by private hospitals (411.3) and other tertiary hospitals (154.4).

In terms of the type of ophthalmic procedures, differences among procedure compositions of EENT Hospital, other tertiary hospitals, secondary hospitals, and private hospitals were significant $(P<0.0001$, Chi-square analysis). Generally, cataract surgery was the most common (44.3\%), followed by small incision lenticule extraction (SMILE, 12.0\%) and vitreoretinal surgery (7.6\%). At the EENT Hospital, SMILE was the most frequent procedure $(25.8 \%)$, followed by cataract surgery $(22.2 \%)$, antivascular endothelial growth factor injection (11.3\%), lacrimal duct reconstruction (11.0\%) and vitreoretinal surgery $(10.2 \%)$. At private hospitals, cataract surgery accounted for $70.2 \%$ of procedures and SMILE accounted for $13.9 \%$ (Fig. 1). At secondary hospitals, the percentage of extraocular procedures, particularly conjunctival surgery such as pterygium surgery $(45.7 \%)$, was much greater than that at tertiary hospitals and private hospitals. Complicated eye surgeries, such as vitreoretinal surgery and corneal transplantation, were mostly performed in tertiary hospitals. Private hospitals performed just $12.2 \%$ of these procedures, even though they had sufficient senior doctors, adequate hospital beds, and comprehensive equipment.

\section{Cataract surgery service}

In 2017, totally 119,264 cataract surgeries were performed in Shanghai, of which EENT Hospital alone contributed $14.2 \%$, the other 26 tertiary hospitals contributed 30.7\%, 46 secondary hospitals contributed $8.5 \%$ and private hospitals contributed $46.6 \%$ (Table 4 ).

Table 3 Ophthalmic services provided at each hospital level

\begin{tabular}{|c|c|c|c|c|}
\hline $\begin{array}{l}\text { Output of } \\
\text { ophthalmic services }\end{array}$ & EENT Hospital & Other tertiary hospitals & Secondary hospitals & Private hospitals \\
\hline \multicolumn{5}{|c|}{ Outpatient and emergency room visit } \\
\hline Local & 374,768 & $1,449,000$ & 984,600 & 315,700 \\
\hline Non-local & 586,175 & 639,400 & 178,200 & 46,300 \\
\hline Total & $960,943(21.0 \%)$ & $2,088,400(45.7 \%)$ & $1,162,800(25.4 \%)$ & $362,000(7.9 \%)$ \\
\hline Average visit & 960,943 & $80,323.1$ & $25,278.3$ & $72,400.0$ \\
\hline \multicolumn{5}{|l|}{ Inpatient admission } \\
\hline Total inpatients & $20,103(14.0 \%)$ & $56,992(39.8 \%)$ & $14,090(9.8 \%)$ & $52,047(36.3 \%)$ \\
\hline Average inpatients & 20,103 & 2192 & 306.3 & $10,409.4$ \\
\hline \multicolumn{5}{|l|}{ Surgical volumes } \\
\hline Total volumes & $72,666(27.4 \%)$ & $87,533(33.0 \%)$ & $25,792(9.7 \%)$ & $79,374(29.9 \%)$ \\
\hline Average volumes & 72,666 & 3366.7 & 560.7 & $15,874.8$ \\
\hline
\end{tabular}

The numbers in brackets represent the percentage of the total number for all hospitals EENT Hospital Eye and Ear, Nose, and Throat Hospital of Fudan University 


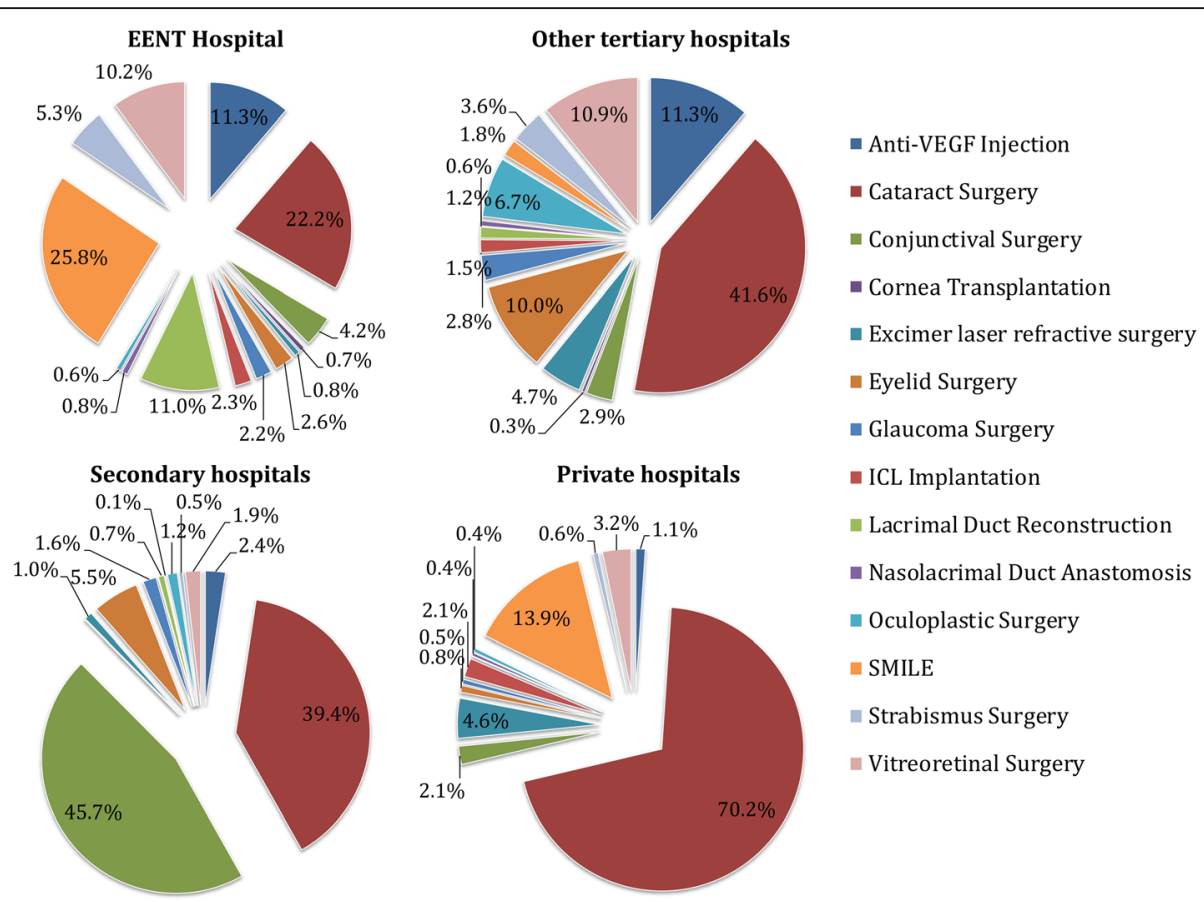

Fig. 1 Comparison of the surgical volume for different subspecialties at each hospital level. EENT Hospital: Eye and Ear, Nose, and Throat Hospital of Fudan University; ICL: implantable collamer lens; SMILE: small incision lenticule extraction

Based on the data provided by Shanghai Municipal Bureau of Statistics, there were around 24.18 million residents in Shanghai in 2017. The cataract surgical rate (cataract operations done per million populations per year) might be around $4932.3(119,264 / 24.18)$ in 2017 in Shanghai. However, given that the total number of cataract surgeries included a certain proportion of surgeries performed on non-residents, this number was higher than the 4251 reported by the National Cataract Surgery Information Reporting System.

At the EENT Hospital, only $43.9 \%$ of cataract procedures were performed as inpatient surgery. This value increased to 76.9 and $80.3 \%$ at secondary and private hospitals, respectively. The mean wait time for cataract surgery ranged from 0.3 to 8 weeks. The daily number of cataract surgical procedures ranged between 20 and 80 at private hospitals, 68 at the EENT Hospital, and $20-65$ at other tertiary hospitals. On average, the annual surgical volume per cataract surgeon was greatest at private hospitals (2136.1), followed by the EENT hospital (1306.3), and other tertiary hospitals (359.1).

Patient selection for cataract surgery showed clear trends among hospitals. The proportion of complicated cataractous patients varied significantly among EENT Hospital, other tertiary hospitals, secondary hospitals, and private hospitals $(P<0.0001$, Chi-square analysis). At the EENT Hospital, $58.0 \%$ of cataract procedures were for complicated cataracts such as highly myopic cataract, traumatic cataract, and congenital cataract, requiring multi-subspecialty eye care, significantly higher than other hospitals on an average level. By comparison, this percentage was only $31.7 \%$ at private hospitals despite the large volume of cataract surgery (Table 4).

Regarding surgical techniques, standard phacoemulsification was the predominant cataract surgical technique (mostly > 95\%). Of all femtosecond laser-assisted cataract surgical procedures (FLACS), 83\% (1358) and 15\% (251) were performed at private hospitals and the EENT Hospital, respectively. Meanwhile, the EENT Hospital performed $33.0 \%$ (325) of more difficult cataract procedures, such as scleral fixation of an intraocular lens (IOL) (Table 4). The proportion of complicated cataract surgery in the EENT Hospital was the highest (7.7\%), followed by other tertiary hospitals $(4.3 \%)$, secondary hospitals $(2.7 \%)$, and private hospitals $(1.7 \%)(P<0.0001$, Chi-square analysis).

A greater number of advanced IOLs, such as toric IOLs and multifocal IOLs, were implanted at the EENT Hospital (386), followed by private hospitals (mean 363.6), accounting for 2.4 and $3.3 \%$ of all IOL implanted at these hospitals. However, capsular tension ring (CTR) and modified CTR implantation were predominantly used at the EENT Hospital (74.1\%, Table 4), followed by other tertiary hospitals (20.5\%) and private hospitals (5.2\%). 
Table 4 Cataract surgical procedures provided at each hospital level

\begin{tabular}{|c|c|c|c|c|c|}
\hline Data on cataract surgery & EENT Hospital & Other tertiary hospitals & Secondary hospitals & Private hospitals & Total \\
\hline \multicolumn{6}{|l|}{ Cataract type } \\
\hline Age-related cataract & 7132 (42.0\%) & 20,747 (56.6\%) & $6959(68.8 \%)$ & $37,949(68.3 \%)$ & 72,787 (61.0\%) \\
\hline Highly myopic cataract & $5604(33.0 \%)$ & 8494 (23.2\%) & $1346(13.3 \%)$ & 9654 (17.4\%) & $25,098(21.0 \%)$ \\
\hline Diabetic cataract & 2377 (14.0\%) & $5362(14.6 \%)$ & $1428(14.1 \%)$ & $7214(13.0 \%)$ & $16,381(13.7 \%)$ \\
\hline Traumatic cataract & $672(4.0 \%)$ & $938(2.6 \%)$ & $66(0.7 \%)$ & $29(0.1 \%)$ & $1705(1.4 \%)$ \\
\hline Congenial cataract & $664(3.9 \%)$ & $845(2.3 \%)$ & $67(0.7 \%)$ & $28(0.1 \%)$ & $1604(1.3 \%)$ \\
\hline Others & $533(3.1 \%)$ & $244(0.7 \%)$ & $248(2.5 \%)$ & $664(1.2 \%)$ & $1689(1.4 \%)$ \\
\hline Total & $16,982(14.2 \%)$ & $36,630(30.7 \%)$ & $10,114(8.5 \%)$ & $55,538(46.6 \%)$ & 119,264 \\
\hline \multicolumn{6}{|c|}{ Surgical techniques used in cataract surgery } \\
\hline Standard phacoemulsification & $15,425(90.8 \%)$ & $35,019(95.6 \%)$ & 9840 (97.3\%) & $53,216(95.8 \%)$ & $113,500(95.2 \%)$ \\
\hline FLACS & $251(1.5 \%)$ & $28(0.1 \%)$ & $0(0.0 \%)$ & $1358(2.4 \%)$ & $1637(1.4 \%)$ \\
\hline Extracapsular cataract extraction & $130(0.8)$ & $334(0.9 \%)$ & $238(2.4 \%)$ & $316(0.6 \%)$ & $1018(0.9 \%)$ \\
\hline Intracapsular cataract extraction & $15(0.1 \%)$ & $19(0.1 \%)$ & $0(0.0 \%)$ & $2(0.0 \%)$ & $36(0.0 \%)$ \\
\hline Scleral fixation of $\mathrm{IOL}$ & $325(1.9 \%)$ & $305(0.8 \%)$ & $36(0.4 \%)$ & $320(0.6 \%)$ & $986(0.8 \%)$ \\
\hline Others & $836(4.9 \%)$ & $925(2.5 \%)$ & $0(0.0 \%)$ & $326(0.6 \%)$ & $2087(1.7 \%)$ \\
\hline Total & $16,982(14.2 \%)$ & $36,630(30.7 \%)$ & $10,114(8.5 \%)$ & $55,538(46.6 \%)$ & 119,264 \\
\hline \multicolumn{6}{|l|}{ IOL used in cataract surgery } \\
\hline Monofocal IOL & $15,866(97.6 \%)$ & $35,023(98.1 \%)$ & 9891 (99.9\%) & $53,322(96.7 \%)$ & $114,102(97.5 \%)$ \\
\hline Multifocal IOL & $247(1.5 \%)$ & $379(1.1 \%)$ & $11(0.1 \%)$ & $1064(1.9 \%)$ & $1701(1.5 \%)$ \\
\hline Toric IOL & 139 (0.9\%) & $276(0.8 \%)$ & $1(0.0 \%)$ & $722(1.3 \%)$ & $1138(1.0 \%)$ \\
\hline Toric multifocal IOL & $0(0.0 \%)$ & $19(0.1 \%)$ & $0(0.0 \%)$ & $32(0.1 \%)$ & $51(0.0 \%)$ \\
\hline Total & $16,252(13.9 \%)$ & 35,697 (30.5\%) & $9903(8.5 \%)$ & $55,140(47.1 \%)$ & 116,992 \\
\hline \multicolumn{6}{|l|}{ CTR used in cataract surgery } \\
\hline CTR & $228(51.7 \%)$ & $93(76.2 \%)$ & $1(100.0 \%)$ & $26(83.9 \%)$ & $348(58.5 \%)$ \\
\hline Modified CTR & $213(48.3 \%)$ & $29(23.8 \%)$ & $0(0.0 \%)$ & $5(16.1 \%)$ & $247(41.5 \%)$ \\
\hline Total & $441(74.1 \%)$ & $122(20.5 \%)$ & $1(0.2 \%)$ & $31(5.2 \%)$ & 595 \\
\hline
\end{tabular}

The numbers in brackets represent the percentage of the total number of procedures performed at each hospital level. The numbers in brackets in the total row represent the percentage of the total number of procedures performed at all hospitals EENT Hospital Eye and Ear, Nose, and Throat Hospital of Fudan University, FLACS femtosecond laser-assisted cataract surgery, IOL intraocular lens, CTR capsular tension ring

\section{Discussion}

This paper provides detailed information about eye care providers in Shanghai in 2017, with the aim to understand the availability and productivity of ophthalmic services and to provide a foundation for quality control of ophthalmic care in the future. No government-led electronic questionnaire survey of this kind, enrolling nearly all eye care hospitals in Shanghai, has been performed previously. The ophthalmic services were evaluated in terms of the basic characteristics of the hospitals, as well as their service output and efficiency. The survey had a particular emphasis on cataract surgery to investigate differences in its provision, independently of the disparity in productivity among different hospitals.

With the revitalization of privatization in China, the number of private healthcare providers has expanded greatly in recent years [12]. The creation of the China Pilot Free-Trade Zone (Shanghai) in late 2013 provided an effective platform for foreign direct investment into the healthcare sector. According to the Annual Report on the National Health Service in China 2017, published by the National Health Commission of the People's Republic of China, the number of patient visits to private hospitals increased by $17.0 \%$ in 2017 versus that in 2016, as compared with $4.2 \%$ in public hospitals. Moreover, China's Health Statistics Yearbook 2018 reported that by the end of 2017, private providers accounted for nearly $50 \%(447,160 / 986,649)$ of all healthcare institutions in China [8]. Shanghai, the most economically developed city in China, has attracted significant private investment in healthcare and has seen a remarkable increase in the number of private hospitals in recent years. 
Our findings capture some typical features of the public and private ophthalmic services in Shanghai. Most hospitals offering ophthalmic services were still public, similar to the characteristics of other provinces of China, such as Guangdong [13]. This was possibly due to historical reasons. Before the implementation of supporting policy and the revitalization of privatization, there were hardly any standard private sectors in realm of healthcare. Thus, patients tend to trust more in the reliability of public hospitals. However, in terms of the basic characteristics of the hospitals, the private sector has now greatly developed to an extent comparable with that of public hospitals of the highest accreditation. Similar to the medical systems of other countries, the private clinics tended to optimize non-clinical factors, such as facilities and wait times, more than public providers [14]. Social investment in private hospitals allowed them to employ many reputable doctors, construct largerscale hospitals, and procure up-to-date equipment to attract patients $[13,15,16]$. This may help explain why the five private hospitals in Shanghai were better equipped than most of the public hospitals in Shanghai. Nevertheless, the EENT Hospital was still the best equipped with the highest percentage of senior doctors, the most beds and highest availability of almost all commercially available equipment for ophthalmic examinations and procedures.

Although there were gaps in the objective characteristics between hospital levels, the disparity in productivity was more concerning. The productive efficiency of hospitals is crucial to their future development, as a guarantee of their potential to meet the ever-increasing demand for healthcare. We found that $20.7 \%$ of the annual outpatient and ER visits were served by $14.8 \%$ of eye doctors at the EENT Hospital. In contrast, private hospitals contributed to just $7.8 \%$ of the total volume, despite employing $16.2 \%$ of the registered workforce, indicating an obvious disparity in technical efficiency. Similar findings were identified in terms of inpatient admissions and surgical volumes among the levels of hospitals. The turnover rate at the EENT Hospital was almost two to three times higher than that at other tertiary and secondary hospitals. Moreover, the EENT Hospital performed $27.4 \%$ of all ophthalmic procedures in Shanghai, whereas the average volume at other hospitals was less than a quarter of this number.

Among the five private hospitals, the surgical volume per doctor was very close to that at the EENT Hospital, which may suggest a much greater efficiency at private hospitals than at most public hospitals. Nevertheless, the difficulty levels of eye care differed markedly among these hospitals. At the EENT Hospital, vitreoretinal surgery, one of the most complicated ophthalmic procedures, accounted for $10.2 \%$ of its surgical volume. A similar value of $10.9 \%$ was found at other tertiary hospitals, but this dropped to $1.9 \%$ at secondary hospitals, due to their lower technical level. Meanwhile, this value was also low, 3.2\%, at private hospitals despite their extensive staff and facilities.

Private hospitals performed $29.9 \%$ of the total surgical volume in Shanghai, but $70.2 \%$ of this was due to cataract surgery. One possible explanation is that the accumulated experience at tertiary hospitals, and greater support from local government and affiliating universities, mean that their professional capabilities had reached a level similar to that in most developed countries [17], and they were able to provide comprehensive eye care across multiple subspecialties. However, unlike the social objectives of public hospitals, the private hospitals tended to provide services with a high profit margin. Cataract surgery and refractive surgery, two highly cost-effective ophthalmic procedures, have a huge potential market considering cataract and refractive error are the two major causes of visual impairment worldwide.

The types of cataract procedures also demonstrate the clear distinction between high-volume public hospitals and private hospitals. Advanced cataract techniques, including FLACS and implantation of advanced IOLs, were mostly performed at the EENT Hospital and private hospitals. However, private hospitals tended to perform fewer procedures in patients with complicated cataracts and performed fewer complex surgical techniques such as scleral fixation or implantation of CTR. Despite this, the private hospitals still provide an important aspect of eye care in Shanghai. The five major high-volume private hospitals performed over $46 \%$ of all cataract surgeries in 2017 in Shanghai, and contributed to the increase in the number of cataract surgeries from 2313 in 2012 to 4251 in 2017. Accordingly, private hospitals helped alleviate the imbalance between medical supply and demand, accelerating the achievement of universal coverage planned in China's new round of healthcare reform. Nevertheless, appropriate supervision and management by the government is necessary to ensure standardization of healthcare services and to reduce the profit-driven focus in healthcare.

Collectively, it is not difficult to perceive the high output and productivity of the EENT Hospital for providing comprehensive eye care to people in Shanghai. The EENT Hospital, alone, provided nearly one fifth of eye care services in Shanghai. It also treated a huge number of non-local patients. However, this high productivity could be a double-edged sword as it allowed easier access to high-quality ophthalmic care to more patients, but this overload may overburden the doctors and impair the quality of the service they provide [18]. 
The high productivity at a single hospital may be due to a combination of internal and external factors. Owing to its large scale, professional staffing and the most comprehensive equipment, the EENT Hospital is ranked second nationally in the field of ophthalmology. These features mean it attracts many patients from neighboring provinces, or further field in China [19]. Another explanation may relate to an ineffective referral triage process. Unlike the process of seeing a doctor in an established medical system, such as in the United States, patients in China do not need to obtain a referral from a family doctor and can bypass the primary healthcare system. Patients flocking to large tertiary hospitals, whether necessary or not, forced the passive increase in efficiency at these hospitals. The over-influx of patients with minor ailments, which could be treated in primary or secondary hospitals, led to the aggravation of the so-called "three long, one short" problem at these large hospitals [1], comprising long registration times, long wait times, long payment queue times, and short visit times, resulting in the pseudo-proposition of difficult access to health services. Overall, a deficit of ophthalmologists remained in Shanghai, with a density of only 0.49 ophthalmologists per 10,000 population, far below the values of 2.56 in Greece, 1.93 in Switzerland in 2017, and 0.60 in United States in 2014.

Nevertheless, the wait time for a consultation in China was relatively short. In Toronto, patients undergoing diagnostic testing for uveitis waited $>40$ days [20]. In China, some patients with serious diseases might benefit from the current system, but the medical resources in tertiary hospitals were exhausted, and largely wasted at lower-level hospitals. In the long term, only an effective referral triage process could address the seemingly uneven distribution of medical resources.

\section{Conclusion}

We performed a city-wide questionnaire survey that provides an overview of the eye care services available in Shanghai. Notwithstanding the rapid development of private sectors, public hospitals still provide the bulk of ophthalmic services. In the hierarchic public system, the EENT Hospital provided almost one fifth of eye care services in Shanghai. The city's services were largely supplemented by private hospitals, which help meet the growing medical demands for treating common diseases. However, further optimization of the hierarchic medical system with proper policy guidance is still necessary to standardize and improve the efficiency of current ophthalmic services.

\section{Abbreviations}

ER: Emergency room; EENT: Eye and Ear, Nose, and Throat; SMILE: Small incision lenticule extraction; FLACS: Femtosecond laser-assisted cataract surgery; IOL: Intraocular lens; CTR: Capsular tension ring

\section{Supplementary Information}

The online version contains supplementary material available at https://doi. org/10.1186/s12913-021-07048-1.

Additional file 1 .

Acknowledgements

Not applicable.

\section{Authors' contributions}

$X J D, Y D, W W H$, and JHD contributed equally to this work, and are co-first authors. XJD, YD, WWH, and JHD conducted the literature search. XJZ, SST, and YL designed the study. WWH, MJC, PJY, HC, HR, and YF collected the data. YD, WWH, JHD, MJC, PJY, HC, HR, and YF conducted the data interpretation. YD, WWH, and JHD prepared the figure and Tables. YD and XJZ wrote the first draft of the manuscript. SST and $Y L$ revised the manuscript. All authors have read and approved the manuscript.

\section{Funding}

Publication of this article was supported by research grants from the National Natural Science Foundation of the People's Republic of China (81870642, 81970780, 81470613, and 81670835), Shanghai High Myopia Study, the Shanghai Talent Development Fund (201604), the Shanghai Youth Doctor Support Program (2014118), and the Outstanding Youth Medical Talents Program of Shanghai Health and Family Planning Commission (2017YQ011). The funders were not involved in the study design, data collection, data analysis, data interpretation or preparation of the manuscript.

Availability of data and materials

The data are available from the authors upon reasonable request and with permission from the Shanghai Medical Quality Control Management Center.

\section{Declarations}

Ethics approval and consent to participate

Our study was reviewed and deemed exempt from formal ethics approval or consent forms by the Ethics Committee at the EENT Hospital of Fudan University.

Consent for publication

Not applicable.

Competing interests

The authors declare that they have no competing interests.

Received: 22 October 2020 Accepted: 17 September 2021

Published online: 02 October 2021

\section{References}

1. Dai J, Wang X, Ayala FJ. Medical informatics and the "three long, one short" problem of large urban hospitals in China. JAMA. 2016;316(3):269-70.

2. Yip W, Fu H, Chen AT, Zhai T, Jian W, Xu R, et al. 10 years of health-care reform in China: progress and gaps in Universal Health Coverage. Lancet (London, England). 2019;394(10204):1192-204.

3. Wang Y, Li X, Zhou M, Luo S, Liang J, Liddell CA, et al. Under-5 mortality in 2851 Chinese counties, 1996-2012: a subnational assessment of achieving MDG 4 goals in China. Lancet (London, England). 2016;387(10015):273-83.

4. Yip WC, Hsiao W, Meng Q, Chen W, Sun X. Realignment of incentives for health-care providers in China. Lancet (London, England). 2010;375(9720): 1120-30.

5. Lancet T. China's health-care reform: an independent evaluation. Lancet (London, England). 2019;394(10204):1113.

6. Meng Q, Liu X, Shi J. Comparing the services and quality of private and public clinics in rural China. Health Policy Plan. 2000;15(4):349-56. 
7. Pan J, Zhao H, Wang X, Shi X. Assessing spatial access to public and private hospitals in Sichuan, China: The influence of the private sector on the healthcare geography in China. Soc Sci Med (1982). 2016;170:35-45.

8. Ma X. China's Health Statistics Yearbook 2018. China; 2018. (in Chinese)

9. Zhang JS, Xu L, Wang YX, You QS, Wang JD, Jonas JB. Five-year incidence of age-related cataract and cataract surgery in the adult population of greater Beijing: the Beijing eye study. Ophthalmology. 2011;118(4):711-8.

10. Tang $Y$, Wang $X$, Wang J, Huang W, Gao Y, Luo Y, et al. Prevalence of agerelated cataract and cataract surgery in a Chinese adult population: the Taizhou eye study. Invest Ophthalmol Vis Sci. 2016;57(3):1193-200.

11. Song P, Du Y, Chan KY, Theodoratou E, Rudan I. The national and subnational prevalence and burden of age-related macular degeneration in China. J Glob Health. 2017;7(2):020703.

12. Xu J, Liu G, Deng G, Li L, Xiong X, Basu K. A comparison of outpatient healthcare expenditures between public and private medical institutions in urban China: an instrumental variable approach. Health Econ. 2015;24(3): 270-9.

13. Eggleston K, Lu M, Li C, Wang J, Yang Z, Zhang J, et al. Comparing public and private hospitals in China: evidence from Guangdong. BMC Health Serv Res. 2010;10:76.

14. Tynkkynen LK, Vrangbaek K. Comparing public and private providers: a scoping review of hospital services in Europe. BMC Health Serv Res. 2018; 18(1):141

15. Aggarwal A, Lewis D, Mason M, Purushotham A, Sullivan R, van der Meulen J. Effect of patient choice and hospital competition on service configuration and technology adoption within cancer surgery: a national, populationbased study. Lancet Oncol. 2017;18(11):1445-53.

16. Klein DJ, Brown AD, Detsky AS. Investing wisely in health care capital. JAMA. 2016;316(15):1543-4

17. Zhang X, Beckles GL, Chou CF, Saaddine JB, Wilson MR, Lee PP, et al. Socioeconomic disparity in use of eye care services among US adults with age-related eye diseases: National Health Interview Survey, 2002 and 2008. JAMA Ophthalmol. 2013:131(9):1198-206.

18. Ward NS, Afessa B, Kleinpell R, Tisherman S, Ries M, Howell M, et al. Intensivist/patient ratios in closed ICUs: a statement from the Society of Critical Care Medicine taskforce on ICU staffing. Crit Care Med. 2013;41(2): $638-45$

19. Tang $C, X u$ J, Zhang M. The choice and preference for public-private health care among urban residents in China: evidence from a discrete choice experiment. BMC Health Serv Res. 2016;16(1):580.

20. Felfeli T, Christakis PG, Bakshi NK, Mandelcorn ED, Kohly RP, DerzkoDzulynsky LA. Referral characteristics and wait times for uveitis consultation at academic tertiary care centres in Toronto. Can J Ophthalmol. 2018;53(6): $639-45$

\section{Publisher's Note}

Springer Nature remains neutral with regard to jurisdictional claims in published maps and institutional affiliations.

Ready to submit your research? Choose BMC and benefit from:

- fast, convenient online submission

- thorough peer review by experienced researchers in your field

- rapid publication on acceptance

- support for research data, including large and complex data types

- gold Open Access which fosters wider collaboration and increased citations

- maximum visibility for your research: over $100 \mathrm{M}$ website views per year

At $\mathrm{BMC}$, research is always in progress.

Learn more biomedcentral.com/submissions 